\title{
On the potential role of retinal sheet transplants for sight restoration
}

\author{
Michael Beyeler \\ Department of Psychology \\ Institute for Neuroengineering \\ eScience Institute \\ University of Washington \\ Seattle, WA 98195 \\ mbeyeler@uw.edu
}

\begin{abstract}
Retinal degenerative diseases such as retinitis pigmentosa (RP) and age-related macular degeneration (AMD) are among the leading causes of blindness in the world. Retinal sheet transplants offer a promising alternative approach to current treatment options. Here I summarize the findings of a recent study demonstrating intact visually evoked responses in rat primary visual cortex (V1) following successful transplantation, and discuss the suitability of retinal sheet transplants as a treatment option for severe retinal degeneration.
\end{abstract}

\section{INTRODUCTION}

Retinal degenerative diseases such as retinitis pigmentosa (RP) and age-related macular degeneration (AMD) are among the leading causes of blindness in the world. These diseases are characterized by a progressive loss of photoreceptors and/or retinal pigment epithelium (RPE), leading to severe remodeling of the retinal circuitry [9] and a gradual loss of vision. However, several studies have shown that cells in the inner retina that connect to the brain may remain functional throughout the disease (e.g., [11]). Therefore, if the diseased cells could be bypassed or replaced with new cells that connect to the functional part of the retina, it might be possible to restore vision in affected individuals.

Although there is no cure for these diseases, several treatment options are currently in development. For example, disease progression might be slowed by introducing trophic factors through micronutrient supplements [7], or stopped by correcting mutated genes through gene therapy [8]. However, these interventions have to happen in early stages of the disease, before photoreceptors are irreversibly degenerated. In severe cases of RP and AMD, the only FDA-approved treatment option are retinal prostheses, which aim to evoke neuronal responses in surviving cells through electrical microstimulation [14] - but their success has been limited to date.

Another approach is to replace diseased cells with healthy cells through transplantation. Human fetal tissue has long been identified as a reliable option for transplantation in the central nervous system (including the eye) in several animal models, and is best transplanted in sheet form that contains the RPE [13]. Although it is well established that retinal sheet transplants can restore responses to flashes of light in the superior colliculus, neuronal responses at the level of visual cortex remain poorly understood.

\section{RETINAL SHEET TRANSPLANTS RESTORE VISION IN RATS WITH SEVERE RETINAL DEGENERATION}

To address this issue, Foik et al. [3] recently investigated the capability of fetal retinal sheet transplants to restore vision in pigmented transgenic line- 3 rats, which is a rat model of severe retinal degeneration. Using a custom-made miniature implantation tool (previously described in Seiler and Aramant, 1998), the investigators were able to embed the retinal sheets into the subretinal space between host degenerated retina and RPE, placed closely to the optic disc in the upper visual field ( [3], Figure 1). A transplant was deemed successful if it i) was stable three months post-transplantation, ii) had produced new photoreceptors and rod bipolar cells (as verified in vivo), and iii) was well integrated with the host retina (as verified through histology ex vivo). Following successful transplantation, the authors measured visually evoked responses in primary visual cortex (V1). Cells were first tested for visual responsiveness using flashes of light; then receptive fields were located using drifting gratings. Visual responses of healthy cells were further characterized via contrast, size, orientation, spatial frequency, and temporal frequency tuning. Neuronal responses were then compared to those of control degenerated animals that did not receive transplants, as well as to non-degenerated NIH and Long-Evans rats.

Foik et al. [3] found that the number of visually responsive cells in V1 improved from 9\% (31/342 cells) in control degenerated rats to $56 \%(89 / 158$ cells) in transplanted rats, as compared to $87 \%(75 / 86)$ in non-degenerated rats ( [3], Figure 4). Moreover, visual sensitivity in transplanted rats had improved to the point where orientation, size, and spatial frequency tuning were statistically indifferent from V1 cells in sighted animals [3], Figure 5). On the other hand, temporal frequency tuning and contrast sensitivity did not significantly improve. The authors also noted that most non-responsive cells in transplanted rats were found further from the retinotopic location of the transplant, implying that the degenerated host retina had in this case failed to relay the visual input. Nevertheless, these findings represent an important step towards producing form vision, as orientationselective responses are key to the perception of form and motion in higher visual cortex.

Consistent with previous studies, Foik et al. found trans- 
planted rats to have higher response latencies than sighted rats, for which they gave two possible reasons: retinal signals might either take longer to travel due to the added layer of retina, or take longer to summate due to a weaker level of photoreceptor input. A combination of both processes might potentially explain the altered V1 response properties described above, with longer response latencies possibly affecting V1 temporal frequency tuning, and lower activity levels altering V1 contrast sensitivity.

Using retrograde tracing of V1 connections, Foik et al. went on to show that feedforward input from the lateral geniculate nucleus, connectivity within V1, and feedback from higher visual areas were present in degenerated rats, but overall to a lesser extent than in transplanted and normal rats. Interestingly, long-range connectivity within V1 (beyond $300 \mathrm{~m}$ ) was reduced the most, whereas local connections remained largely unaffected. Furthermore, transplantation was able to restore the circuitry back to a level comparable to normal rats. These findings suggest the presence of an activitydependent plasticity mechanism that may lead to a reduction of cortical connections in the absence of visual input, but can be recruited to restore connectivity even months after vision loss. However, without further analysis it is unclear whether this plasticity could lead to functional cortical reorganization, or whether it mainly resembles the sort of corruptive retinal remodeling that occurs in later stages of photoreceptor disease (for a recent discussion on the subject, see [1]). Improved cortical responses could have also been due to neuroprotection of the remaining host photoreceptors, as the reactive change of Müller glial cells is a well-known obstacle for transplant integration [5]. Although the authors argue that this might not be an issue for fetal tissue sheets, more research is needed to investigate the many roles that Müller cells play in retinal degeneration [4] and elucidate which retinal cells participate in transplant-host connectivity.

In summary, this study offers strong evidence that retinal sheet transplants can lead to detailed responses in visual cortex, suggesting that more complete recovery of V1 function would be possible through transplants of sheets covering more retinal area.

\section{DISCUSSION}

However, an important open question is whether these findings will apply to transplantation in humans. Several clinical trials with retinal sheet transplants have been underway since the late 1990s, but results have been mixed. Early clinical trials reported no adverse effects, but also no lasting vision improvement [6]. The best results were achieved in a Phase II clinical trial conducted in a group of ten patients (six RP and four AMD) where the transplanted retinal sheet included the RPE [10]: seven patients showed visual improvements after one year, with vision remaining the same in one RP patient, and vision decreasing in two others. In one subject, vision improved from 20/800 pre-operative to 20/160 at the 1-year mark, and remained stable at 20/200 over five years. Future research will have to show if these results can be repeated in a larger study sample.
The use of a transplant that includes the RPE is an attractive strategy, as healthy RPE cells should theoretically be able to restore all functions of the degenerated host RPE. However, the success of this strategy strongly depends on cell delivery and maintenance. In the future, it may be possible to differentiate retinal and photoreceptor progenitors directly from embryonic stem cellswithout the need for fetal tissue. To this end, a Phase I trial involving a stem cell-derived RPE patch just recently showed significant vision improvement in two AMD patients [2].

In conclusion, this study represents an essential step towards determining the suitability of retinal sheet transplants as a treatment option for severe retinal degeneration. However, much work remains to be done until this is a viable treatment option in humans. Although availability of fetal tissue will likely remain limited, much could be learned about the cellular mechanisms of sight restoration through this line of research, thus giving hope that the blinding effects of incurable eye diseases could one day be reversed.

\section{REFERENCES}

[1] Beyeler M, Rokem A, Boynton GM, Fine I (2017). "Learning to see again: Biological constraints on cortical plasticity and the implications for sight restoration technologies." J Neural Eng 14:051003.

[2] da Cruz L et al. (2018). "Phase 1 clinical study of an embryonic stem cell-derived retinal pigment epithelium patch in age-related macular degeneration." Nat Biotechnol.

[3] Foik AT, Lean GA, Scholl LR, McLelland BT, Mathur A, Aramant RB, Seiler MJ, Lyon DC (2018). "Detailed visual cortical responses generated by retinal sheet transplants in rats with severe retinal degeneration." J Neurosci:1279-18.

[4] Hippert C, Graca AB, Barber AC, West EL, Smith AJ, Ali RR, Pearson RA (2015). "Müller Glia Activation in Response to Inherited Retinal Degeneration Is Highly Varied and Disease-Specific." PLOS ONE 10:e0120415.

[5] Hippert C, Graca AB, Pearson RA (2016). "Gliosis Can Impede Integration Following Photoreceptor Transplantation into the Diseased Retina." In: Retinal Degenerative Diseases (Bowes Rickman C, LaVail MM, Anderson RE, Grimm C, Hollyfield J, Ash J, eds), pp 579-585, Advances in Experimental Medicine and Biology. Springer International Publishing.

[6] Humayun MS, Juan E de, Cerro M del, Dagnelie G, Radner W, Sadda SR, Cerro C del (2000). "Human Neural Retinal Transplantation." Invest Ophthalmol Vis Sci 41:3100-3106.

[7] Lavail MM (2005). "Survival Factors for Treatment of Retinal Degenerative Disorders: Preclinical Gains and Issues for Translation Into Clinical Studies." Retina 25.

[8] Liu MM, Tuo J, Chan C-C (2011). "Gene therapy for ocular diseases." British Journal of Ophthalmology 95:604-612.

[9] Marc RE, Jones BW, Watt CB, Strettoi E (2003). "Neural remodeling in retinal degeneration." Prog Retin Eye Res 22:607-655.

[10] Radtke ND, Aramant RB, Petry HM, Green PT, Pidwell DJ, Seiler MJ (2008). "Vision improvement in retinal degeneration patients by implantation of retina together with retinal pigment epithelium." $A m J$ Ophthalmol 146:172-182.

[11] Santos A, Humayun MS, de Juan E, Greenburg RJ, Marsh MJ, Klock IB, Milam AH (1997). "Preservation of the inner retina in retinitis pigmentosa. A morphometric analysis." Arch Ophthalmol 115:511-515.

[12] Seiler MJ, Aramant RB (1998). "Intact sheets of fetal retina transplanted to restore damaged rat retinas." Invest Ophthalmol Vis Sci 39:21212131.

[13] Seiler MJ, Aramant RB (2012). "Cell replacement and visual restoration by retinal sheet transplants." Progress in Retinal and Eye Research 31:661-687.

[14] Weiland JD, Walston ST, Humayun MS (2016). "Electrical Stimulation of the Retina to Produce Artificial Vision." Annual Review of Vision Science 2:273-294. 